\title{
Patrones de distribución de felinos silvestres (Carnivora: Felidae) en el trópico seco del Centro-Occidente de México
}

\author{
Juan Felipe Charre-Medellín ${ }^{1}$, Tiberio Cesar Monterrubio-Rico ${ }^{1}$, Daniel Guido-Lemus ${ }^{1}$ \& \\ Eduardo Mendoza ${ }^{2}$ \\ 1. Laboratorio de Ecología de Vertebrados Terrestres Prioritarios, Facultad de Biología, Universidad Michoacana de \\ San Nicolás de Hidalgo. Avenida Francisco J. Mújica S/N, Ciudad Universitaria, Colonia Felícitas del Río, Morelia, \\ Michoacán, 58030, México; jfcharre@yahoo.com.mx, tmonter2002@yahoo.com.mx, guido_chicken@hotmail.com \\ 2. Laboratorio de Análisis para la Conservación de la Biodiversidad, Instituto de Investigaciones sobre los Recursos \\ Naturales, Universidad Michoacana de San Nicolás de Hidalgo. Avenida San Juanito Itzicuaro S/N. Colonia Nueva \\ Esperanza, Morelia, Michoacán, 58337, México; mendoza.mere@gmail.com
}

Recibido 30-VII-2014. Corregido 01-II-2015. Aceptado 03-III-2015.

\begin{abstract}
Distribution patterns of wild felids (Carnivora: Felidae) in the dry tropics of Central-Western Mexico. The Michoacán state is characterized by the existence of important environmental heterogeneity in terms of climate, topography and types of vegetation, which includes the worldwide endangered tropical dry forest. Some reports indicating the presence of the six species of felids occurring in Mexico in this region have been made; however, evidence to support these reports is scant, and filling this lack of information is particularly critical in the case of threatened species or habitats. The aim of this study was to systematize and analyze data distribution patterns of felids in the state of Michoacán, in the Central-Western Mexico. We conducted a review of literature and databases to compile species presence records in the study region. Moreover, we analyzed data obtained from ten years of field work conducted in the region, in which complementary methods (detection of direct and indirect evidence of species occurrence along transects, camera-trapping and interviews to local people) were applied to detect the presence of felid species. We compiled a total of 29 presence records of felids in the region from our review. Additionally, field work, which accumulated $1107.5 \mathrm{~km}$ of walked transects, and 8699 camera-trap days, produced 672 records of species presence. Lynx rufus was the species with the lowest number of records and the most restricted distribution. In contrast, the species with the greatest number of records was Leopardus pardalis $(\mathrm{n}=343)$. In general, $89 \%$ of felids records occurred below 1000 masl. Overall mean annual temperature of presence records was $24{ }^{\circ} \mathrm{C}$ and mean annual precipitation was $1040 \mathrm{~mm}$. The species whose presence records showed the most distinctive pattern, in terms of temperature and precipitation associated, was L. rufus $\left(15.8 \pm 1.3^{\circ} \mathrm{C}\right.$ and $\left.941 \pm 171 \mathrm{~mm}\right)$. Results of a cluster analysis showed that areas supporting different combinations of eco-regions and types of vegetation could be grouped in five clusters having different assemblages of felid species and camera-trapping records. This study results useful to garner a more comprehensive view of the distribution patterns of felids in a region with important environmental contrasts and subjected to an increased human pressure. Moreover, this study provides insights that further our understanding of the relationship between environmental variables and felid distribution patterns which may have an impact for conservation and management strategies at the local and regional levels. Rev. Biol. Trop. 63 (3): 783-797. Epub 2015 September 01.
\end{abstract}

Key words: camera-trapping, environmental heterogeneity, habitat preferences, wild mammal ecology.

Las actividades humanas han reducido el tamaño de las poblaciones de mamíferos silvestres y sus ámbitos de distribución, a nivel global, a tal grado, que han colocado en riesgo de extinción a una cuarta parte del total de las especies de este grupo (Ceballos \& Ehrlich, 2002; Baillie, Griffiths, Turvey, Loh, \& Collen, 2010). En el neotrópico las especies de la familia Felidae son de las más afectadas por las actividades humanas, ya que además de la 
destrucción de su hábitat, están consideradas como una amenaza para el ganado, son vendidas como mascotas y sus pieles son comercializadas (Ceballos, List, Medellín, Bonacic, \& Pacheco, 2010a).

En México habitan seis de las doce especies de felinos presentes en el continente americano: lince (Lynx rufus), puma (Puma concolor), jaguarundi (Puma yagouaroundi), ocelote (Leopardus pardalis), tigrillo o margay (Leopardus wiedii) y el jaguar (Panthera onca) (Ceballos et al., 2010a). Sin embargo, no se cuenta con información precisa y actualizada sobre sus patrones de distribución a nivel local y regional. Asimismo, se desconoce en gran medida el efecto que las variables ambientales tienen sobre sus patrones de distribución (Leopold, 1959; Emmons \& Feer, 1997).

La vertiente del Pacífico es una región de México que en particular carece de información detallada sobre la presencia y distribución de especies de felinos, particularmente en la zona delimitada por el estado de Michoacán. Esto se debe en parte a la dificultad de acceso a esta región, caracterizada por una compleja topografía, consecuencia de la convergencia del Eje Neovolcánico, la Sierra Madre del Sur y la depresión de las cuencas del Balsas y Tepalcatepec (Garduño-Monroy, 2005). De acuerdo con Leopold (1959), las seis especies de felinos presentes en México se distribuían ampliamente en Michoacán. Sin embargo, la información disponible para sustentar esa afirmación era muy limitada y aunque en las décadas siguientes se agregaron nuevos registros de felinos en la región, la información es aún insuficiente, dispersa geográficamente y en algunos casos imprecisa (Brand, 1960; Álvarez, Arroyo-Cabrales, \& González-Escamilla, 1987; Olalde-García, 1997).

La región delimitada por el estado de Michoacán estuvo originalmente dominada por el bosque tropical caducifolio o (selva baja caducifolia), en combinación con el bosque tropical subcaducifolio (selva mediana) en las áreas más húmedas, y bosques templados de encino-pino en elevaciones superiores a los 1500 msnm (Ceballos, García, Salazar, \&
Espinoza, 2010b). Sin embargo, la creciente actividad humana ha provocado que el paisaje de la región se caracterice en la actualidad por la presencia de un complejo mosaico de diferentes tipos de cobertura y usos del suelo, tales como bosques tropicales y bosques templados altamente fragmentados por la agricultura y ganadería (Bocco, Mendoza, \& Masera, 2001). El creciente impacto humano sobre los hábitats naturales existentes en la región aumenta la importancia de llenar el vacío de información existente sobre los patrones actuales de distribución de las especies de felinos.

Por lo anterior, los objetivos de este estudio se centraron en: 1) recabar y analizar la información existente sobre la distribución de las especies de felinos en la Costa del Pacífico, en particular en la región delimitada por el estado de Michoacán; 2) actualizar la información sobre la distribución actual de los felinos, a partir de la integración y análisis de los registros obtenidos para este grupo mediante trabajo de campo sistemático realizado durante los últimos 10 años; y 3) analizar cómo se distribuyen las especies en relación con la existencia de distintas ecorregiones, tipos de vegetación y variaciones climáticas. Todo con el fin de avanzar en el conocimiento de la ecología y estado de conservación de los felinos en el trópico seco del Pacífico Central.

\section{MATERIALES Y MÉTODOS}

Área de estudio: El estado de Michoacán se localiza entre las coordenadas $17^{\circ} 55^{\prime}$ - 202' ' N y $100^{\circ} 04^{\prime}$ - $103^{\circ} 44^{\prime}$ 'W, y abarca una superficie de $58364 \mathrm{~km}^{2}$ (Garduño-Monroy, 2005). Debido a su heterogeneidad topográfica, altitudinal (0 hasta $3569 \mathrm{msnm}$ ) y de tipos de vegetación, en el estado se han identificado las siguientes ecorregiones (Olson et al., 2001; Garduño-Monroy, 2005): Bajío, Eje Neovolcánico, Bajo Balsas, Sierra Madre del Sur y Costa. En la región del Bajío, predomina el matorral subtropical y extensas planicies; en el Eje Neovolcánico, predominan los bosques templados de coníferas y de encino. En las ecorregiones del Bajo Balsas, Costa y Sierra Madre del Sur, 
dominan los bosques tropicales caducifolios y subcaducifolios, con una menor representación de bosques templados típicos de elevaciones superiores (Carranza-González, 2005).

Integración de registros de presencia de felinos en la región de Michoacán a partir de referencias bibliográficas y bases de datos: Se revisaron y consultaron artículos científicos, tesis de grado y bases de datos nacionales como la Red Mundial de Información sobre Biodiversidad REMIB (http://www.conabio.gob.mx/ remib/doctos/remib_esp.html) y la de la Unidad de Informática para la Biodiversidad UNIBIO (http://unibio.unam.mx/). Adicionalmente, también se consultaron portales internacionales como el: Global Biodiversity Information Facility GBIF (http://www.gbif.org/) y Mammal Networked Information System MaNIS (http:// manisnet.org/). Dentro de las fuentes consultadas fueron particularmente importantes los registros de referencia provenientes de los proyectos: "Mamíferos de México", del cual los especímenes fueron depositados en colecciones de Estados Unidos y Canadá (López-Wilchis, 1998), "Biodiversidad de los Mamíferos del Estado de Michoacán” en Álvarez-Solórzano y López-Vidal (1998) y el proyecto "Computarización de las colecciones del museo de Zoología Alfonso L. Herrera para su incorporación a la REMIB: Fase I", en León-Paniagua (1999). Todos los registros que se obtuvieron fueron revisados, verificados y corregidos, en caso de ser necesario, para asegurarse que no hubiera errores en sus coordenadas geográficas e información de localidad de procedencia.

Registros de presencia de especies de felinos obtenidos en campo (2003-2013): A partir del 2003 se inició un programa para el registro de felinos en sitios seleccionados con base en su accesibilidad geográfica, tipo de vegetación, seguridad para el acceso y apoyo de los pobladores locales.

La recolecta de registros sobre la presencia de felinos se realizó a través de: 1) registros directos (i.e., observaciones) e indirectos (i.e., cráneos, otros restos biológicos, excretas y huellas), ambos obtenidos durante recorridos diurnos y nocturnos de transectos; 2) cámaras-trampa, y 3) la realización de entrevistas estructuradas. Los sitios fueron visitados una sola ocasión, con la excepción de algunas localidades ubicadas en la Costa y la Sierra Madre del Sur 2010 y el 2013.

Los transectos tuvieron una longitud de 1.5 a $2.5 \mathrm{~km}$. El 50\% de los recorridos de estos transectos fueron diurnos (6:00 a 10:00hr), el 25\% nocturnos (22:00-2:00hr) y el 25\% restante se realizó durante el crepúsculo (17:00-20:00hr). Las huellas se identificaron utilizando guías de campo y el apoyo de asistentes locales con experiencia (Aranda, 2000). Los cráneos encontrados se depositaron en las colecciones de mamíferos del Museo de Zoología "Alfonso L. Herrera" de la Facultad de Ciencias de la Universidad Nacional Autónoma de México y en la Facultad de Biología de la Universidad Michoacana de San Nicolás de Hidalgo para que fueran catalogados.

Las cámaras-trampa se colocaron al borde de senderos, caminos y manantiales con el fin de maximizar la probabilidad de registrar la presencia de felinos (Silver et al., 2004). Este muestreo, se realizó principalmente después de la época de lluvias (noviembre) y durante la época de secas (diciembre a mayo). Se utilizaron los siguientes modelos de cámarastrampa: Moultrie Game Spy, 4.0 megapixels (Moultrie feeders, Inc. Alabaster, Alabama) y Wildview Xtreme 4 y 5 (Wildview Co., Grand Prairie, Texas). Se consideraron como registros independientes los obtenidos para la misma especie y estación de cámara-trampa pero obtenidas con una diferencia mínima de 24 horas o cuando se registró más de un individuo en una fotografía (Botello, Monroy, Illoldi-Rangel, Trujillo-Bolio, \& Sánchez-Cordero, 2007; Monroy-Vilchis, Zarco-González, RodríguezSoto, Soria-Díaz, \& Urios, 2011).

Para identificar los felinos registrados por fotografías (Fig. 1), se empleó la guía para mamíferos del Neotrópico de Emmons y Feer (1997). Además, para diferenciar especies similares de felinos, como L. wiedii y L. pardalis se 

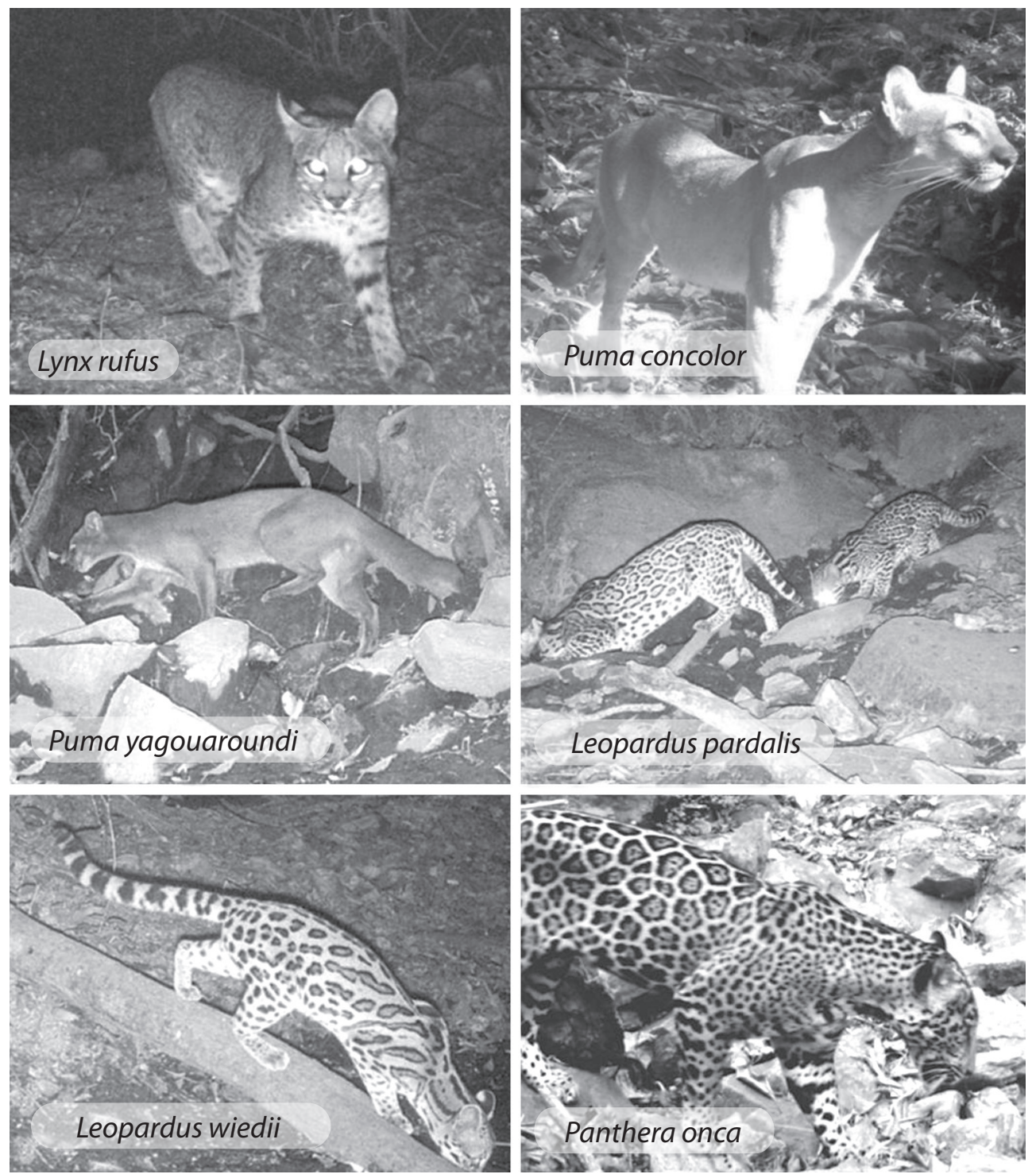

Fig. 1. Felinos silvestres registrados mediante cámaras-trampa en la región de estudio.

Fig. 1. Species of wild felids recorded during the camera-trapping survey made for this study.

consultó respectivamente a Murray y Gardner (1997) y Oliveira (1998).

Las entrevistas se aplicaron a los habitantes de las localidades muestreadas y sirvieron principalmente para identificar áreas con presencia de los felinos, las cuales fueron visitadas para registrar sus coordenadas. En ocasiones las entrevistas también sirvieron para obtener evidencia, tal como la piel de felinos cazados, que sirvió para confirmar la presencia de las especies.

Todos los registros bibliográficos y los obtenidos en campo durante este trabajo, están disponibles para quien los requiera a petición expresa dirigida al primer autor de este trabajo.

Análisis de los patrones de distribución de las especies de felinos: Una vez que las 
coordenadas de todos los registros de felinos se verificaron, se incorporaron a un sistema de información geográfica (SIG) creado con el programa ArcGis versión 9.3 (ESRI, 2008). En este SIG también se incorporaron los mapas de las ecorregiones propuestas por Olson et al. (2001) y de la vegetación y uso de suelo a escala 1:250 000 (INEGI, 2013a), así como los polígonos de las áreas naturales protegidas de carácter estatal y federal (CONANP, 2013). En el caso de la cartografía de vegetación y uso del suelo se combinaron las siguientes categorías: pastizales inducidos, pastizales cultivados, agricultura de temporal y agricultura de riego, para formar una nueva categoría que se denominó: Usos Agropecuarios. Finalmente, al SIG se le incorporaron las siguientes capas de información en formato raster: modelo digital de elevación (MDE) con una resolución de 30 metros por pixel (INEGI, 2013b), y variables bioclimáticas relacionadas con la precipitación y temperatura que se descargaron del sitio web WorldClim-Global Climate Data (www. worldclim.org). En específico se incorporaron las siguientes variables bioclimáticas: $\mathrm{BIO} 1=$ temperatura media anual, $\mathrm{BIO}=$ temperatura máxima de mes más caluroso, $\mathrm{BIO6}=$ temperatura mínima de mes más frío, $\mathrm{BIO} 12=$ precipitación anual, $\mathrm{BIO} 13$ = precipitación de mes más húmedo y $\mathrm{BIO} 14$ = precipitación de mes más seco. Estas variables han sido asociadas con la distribución de los felinos en otras regiones (Jiménez-Maldonado, 2007; Bárcenas \& Medellín, 2010; Cuervo-Robayo \& MonroyVilchis, 2012).

Para evaluar si existía una distribución diferencial de las especies de felinos en función de las características del ambiente, se realizó un análisis de escalamiento multidimensional no métrico (NMDS). Se usó el comando vegdist de la biblioteca Vegan del programa R (R Core Team, 2013) para calcular las distancias euclidianas entre los registros de las especies en un espacio multivariado definido por las variables climáticas antes mencionadas y la elevación. Posteriormente, usamos el comando MetaMDS para realizar el análisis de NMDS.
Este comando transforma automáticamente los datos buscando mejorar la calidad de la ordenación y busca de manera iterativa la solución del análisis con menor estrés (i.e., donde la ordenación representa de manera más fiel las distancias originales en el espacio multivariado) (Borcard, Gillet, \& Legendre, 2011).

Por último, se realizó un análisis de conglomerados para determinar si distintas áreas dentro de la región de estudio, representadas a partir de distintas combinaciones de ecorregiones y tipos de vegetación, se agrupaban en función de los patrones de presencia y tasas de captura de las especies de felinos. Se asignaron las siguientes claves de identificación a las ecorregiones (E1 Bajío, E2 Eje Neovolcánico, E3 Bajo Balsas, E4 Sierra Madre del Sur y E5 Costa) y tipos de vegetación (V1 bosque tropical caducifolio, V2 bosque tropical subcaducifolio, V3 bosque de pino, V4 bosque de pino-encino, V5 bosque de encino, V6 área agropecuaria). Posteriormente, se calcularon las tasas de captura (no. de registros/100 días cámara-trampa) de cada especie para cada combinación ecorregión-tipo de vegetación. Con base en estos datos se calculó la distancia euclidiana entre las distintas áreas, usando el comando vegdist de la biblioteca Vegan del programa R (R Core Team, 2013). Se transformaron los datos (normalización) con la función decostand de Vegan. Se probaron distintas técnicas de aglomeramiento jerárquico para obtener un dendrograma incluyendo: ligamiento sencillo, ligamiento completo, UPGMA y Ward (para consultar detalles de estas técnicas ver Borcard et al., 2011). Para evaluar cuál de los dendrogramas resultantes era más efectivo para representar las distancias euclideanas entre las distintas áreas, se realizaron correlaciones cofenéticas (Borcard et al., 2011). Para determinar el número óptimo de conglomerados en el dendrograma se utilizó el comando silhouette de R (Borcard et al., 2011). Para este análisis sólo utilizamos los registros de cámaras-trampa por ser los más directamente comparables entre especies. 


\section{RESULTADOS}

Registros bibliográficos: Se reunieron 29 registros de los felinos (Fig. 2): P. onca (n $=13)$, L. rufus $(\mathrm{n}=4), P$. yagouaroundi $(\mathrm{n}=$ 4), L. wiedii $(\mathrm{n}=4), P$. concolor $(\mathrm{n}=2)$ y $L$. pardalis $(\mathrm{n}=2)$. Sin embargo, la mayoría de los registros carecían de una adecuada información que permitiera establecer de manera precisa sus coordenadas de origen.

Las fechas de los registros de origen bibliográfico, abarcaron desde el lince recolectado

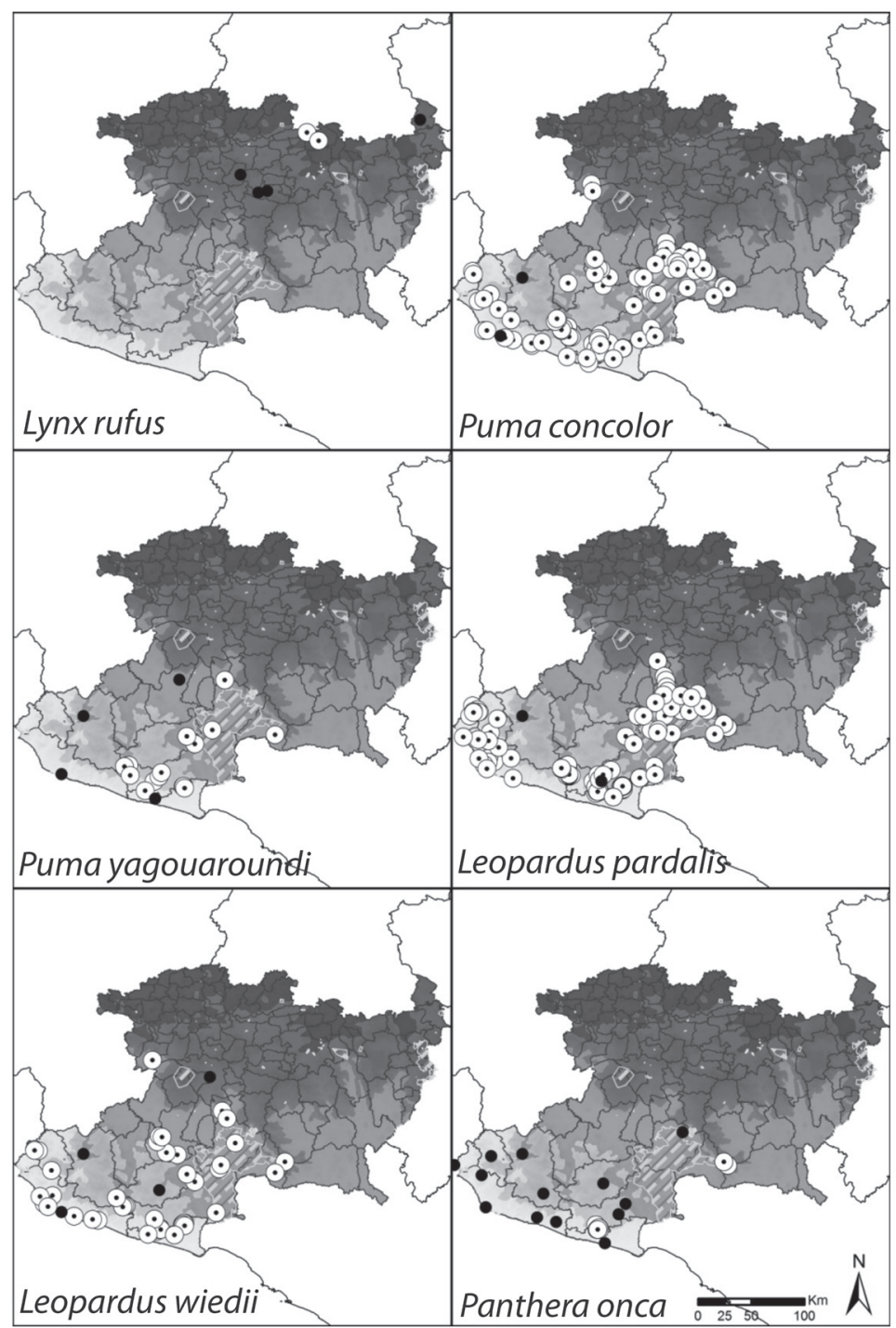

Fig. 2. Distribución geográfica de los registros de felinos obtenidos en este estudio. Los círculos negros corresponden a registros bibliográficos y los círculos blancos a registros obtenidos durante el trabajo de campo reportado en este estudio. La escala de grises representa las cinco ecorregiones del estado, de norte a sur; Bajío, Eje Neovolcánico, Bajo Balsas, Sierra Madre del Sur y Costa.

Fig. 2. Geographic distribution of felid species records in the study area. Black dots correspond to records obtained from literature and data bases whereas white dots were obtained during field work conducted for this study. The gray scale represents the five ecoregions occurring in the state, from north to south; Bajio, Eje Neovolcánico, Bajo Balsas, Sierra Madre del Sur and Costa. 
por el "Smithsonian Institution" en el municipio de Pátzcuaro en 1892 (López-Wilchis, 1998), hasta la fotografía del tigrillo obtenida mediante una cámara-trampa en el municipio de Uruapan en 2005 (Chávez-León \& Zaragoza-Ribera, 2009).

Fueron muy poco los casos en los que se contó con especímenes recolectados que estuvieran depositados en colecciones científicas. En ese sentido, destacan tres registros de L. rufus (López-Wilchis, 1998), uno de $P$. yagouaroundi (Álvarez-Solórzano \& LópezVidal, 1998), y uno de L. wiedii (León-Paniagua, 1999).

La mayoría de los 29 registros bibliográficos correspondieron a reportes circunstanciales. Por ejemplo, se encontraron 13 registros bibliográficos de $P$. onca, que carecían de una precisa georreferenciación de respaldo, una condición que dificultó la verificación o confirmación de los registros (Núñez, 2007; 2012). Entre los registros destacan por su ubicación los reportados por Brand (1960), con coordenadas correspondientes a la cabecera municipal de Coalcomán, así como el jaguar identificado en 1987, por medio de una piel encontrada en una curtiembre del municipio de Arteaga (Núñez, 2012).

Del total de estos registros, diez se presentaron en zonas urbanas, nueve en áreas de usos agropecuarios, tres en bosques de pino, tres en bosque de pino-encino, tres en bosque tropical subcaducifolio y finalmente uno en bosque tropical caducifolio. Con respecto a las ecorregiones, no se encontraron registros bibliográficos para el Bajío (Fig. 2). En contraste, en la ecorregión Costa, se concentraron once registros. El 55\% de los registros se encontraron a una altura por debajo de $1000 \mathrm{msnm}$.

Registros obtenidos en campo: Se acumuló un esfuerzo de muestreo de $1107.5 \mathrm{~km}$ de transectos y 8699 días/cámara-trampa, que produjeron un total de 672 registros de felinos en 302 localidades distintas (Fig. 2). Se obtuvieron registros con todos los métodos (Cuadro 1), aunque el más eficiente fue el uso de cámaras-trampa, que generó el $74.3 \%$ de los registros, seguido por la identificación de huellas $(11.3 \%)$ y las entrevistas $(9.5 \%)$. La especie con el mayor número de registros totales fue $L$. pardalis $(51.1 \%)$, seguida de $P$. concolor (29.8\%). En contraste, L. rufus fue la especie con el menor número de registros (0.4\%). Con respecto a los registros obtenidos mediante cámaras-trampa, el ocelote presentó la tasa de captura más alta con 29.8 registros por 100 días/cámara-trampa, seguido del puma con 14.9, tigrillo con 10.7, jaguarundi con 9.8, jaguar con 3.6 y finalmente el lince con 3.3.

Se recolectaron cinco cráneos que se depositaron en colecciones científicas (Cuadro 1). Estos cráneos correspondieron a las siguientes especies: L. pardalis (MZFC 8888), P. concolor (MZFC 8897), P. yagouaroundi (MZFC 8890 y UMSNH 3413) y P. onca (MZFC 11063).

Patrones de distribución de las especies de felinos: La escasez de registros de felinos obtenidos a partir de la revisión de bibliografía

CUADRO 1

Registros de felinos por método de muestreo

TABLE 1

Number of records per species and surveying method

\begin{tabular}{lccccccc}
\multicolumn{1}{c}{ Tipos de evidencia } & L. rufus & P. concolor & P. yagouaroundi & L. pardalis & L. wiedii & P. onca & Total \\
Avistamiento & 0 & 6 & 1 & 10 & 4 & 0 & 21 \\
Fotografía & 2 & 128 & 15 & 279 & 54 & 21 & 499 \\
Cráneos & 0 & 1 & 2 & 1 & 0 & 1 & 5 \\
Entrevistas & 0 & 25 & 4 & 19 & 16 & 0 & 64 \\
Excretas & 0 & 3 & 0 & 0 & 0 & 0 & 3 \\
Huellas & 0 & 36 & 0 & 33 & 7 & 0 & 76 \\
Pieles & 1 & 1 & 0 & 1 & 1 & 0 & 4 \\
Total & 3 & 200 & 22 & 343 & 82 & 22 & 672 \\
\hline
\end{tabular}


y de bases de datos limitó la posibilidad de hacer una descripción detallada de la distribución de las especies de felinos. Resalta, sin embargo, el que $65 \%$ de estos registros, corresponden a sitios que actualmente están sujetos a usos agropecuarios o son zonas urbanas.

Cuando se analizaron en conjunto todos los registros, destacaron el puma, ocelote y tigrillo como las especies con una distribución más amplia, que incluyó las ecorregiones de Bajo Balsas, la Sierra Madre del Sur y Costa. En estas regiones se registró en menor medida al jaguar y jaguarundi. Sin embargo, fue la ecorregión de la Sierra Madre del Sur donde se encontró el mayor número de registros, $(\mathrm{n}=$ 271). En contraste, la ecorregión del Bajío es donde se presentó el menor número de registros ( $\mathrm{n}=3$ ), que sólo incluyeron a L. rufus (Fig. 2).

En general, los registros de felinos se distribuyeron en un gradiente altitudinal entre los 3 y 2746 msnm. Sin embargo, un 89.5\% de los registros se localizó por debajo de los 1000 msnm. L. rufus fue la especie cuyos registros tuvieron una distribución altitudinal más restringida ya que sólo se encontraron entre los 1700 y $2700 \mathrm{msnm}$. En contraste, $P$. concolor y $L$. wiedii fueron las especies que se registraron en el intervalo altitudinal más amplio (3 a $1758 \mathrm{msnm}$ ).

El clima de la región donde se localizaron los 701 especímenes de felinos, presentó una precipitación durante el mes más seco de menos de $10 \mathrm{~mm}$, y el 82\% $(\mathrm{n}=579)$ de los registros, correspondieron a zonas con escasa precipitación, pero con una precipitación en el mes más húmedo entre 201 y $300 \mathrm{~mm}$. El $56.0 \%(\mathrm{n}=392)$ de los registros se presentaron en áreas con una temperatura promedio anual entre 21 y $25^{\circ} \mathrm{C}$. El $71 \%(\mathrm{n}=496)$ de los registros se localizaron en áreas donde la temperatura del mes más cálido fluctuó entre los 31 y $35^{\circ} \mathrm{C}$. Por otra parte, el $69 \%(n=483)$ de los registros, se presentó en áreas donde la temperatura más fría varió entre 16 y $20^{\circ} \mathrm{C}$. En la mayoría de especies, a excepción del lince y el jaguar, los registros se presentaron en localidades donde la temperatura máxima fue superior a los $39^{\circ} \mathrm{C}$.
En general, los felinos se registraron en sitios que presentaron una temperatura media anual de $24{ }^{\circ} \mathrm{C}$ y una precipitación anual de 1 $040 \mathrm{~mm}$. La única excepción fue L. rufus, que se registró a una temperatura promedio de 15.8 $\pm 1.3{ }^{\circ} \mathrm{C}$ y una precipitación media anual de $941 \pm 171 \mathrm{~mm}$ (Apéndice 1).

Los resultados del análisis de NMDS hicieron evidente que la mayoría de las especies de felinos presentaron una afinidad con respecto a las condiciones ambientales de las localidades en las que hubo evidencia de su presencia (Fig. 3). La única excepción fue el lince, ya que las localidades donde se presentó se separaron claramente en el NMDS en particular por estar ubicadas a una mayor altitud y una precipitación del mes más seco, superior a los $6 \mathrm{~mm}$ (Fig. 3).

$\mathrm{Al}$ analizar la tasa de registro de los felinos en las cámaras-trampa, encontramos que en la ecorregión del Bajío solo se registró al lince con una tasa de captura de 3.3 registros por 100 días/cámara-trampa. Mientras que en el bosque tropical caducifolio de la Costa y en los bosques de pino-encino de la Sierra Madre del Sur se registraron la mayor cantidad de especies de felinos con cinco y cuatro y las mayores tasas de captura de felinos con 9 y 8.5 registros por 100 días/cámara-trampa, respectivamente.

De acuerdo con el dendrograma resultante del análisis de conglomerados de las combinaciones de ecorregiones y tipos de vegetación se logró distinguir cinco grupos (Fig. 4A). El grupo uno, incluyó a cinco áreas dentro de las ecorregiones del Eje Neovolcánico, Bajo Balsas y Costa, donde se registraron a cinco especies de felinos (sólo el lince estuvo ausente), con una tasa de captura de 15.3 registros por 100 días/cámara-trampa para los felinos registrados en bosques tropicales caducifolios, 3.8 registros por 100 días/cámara-trampa para los felinos registrados en bosque tropical subcaducifolio, y 13.7 registros por 100 días/ cámara-trampa para los felinos registrados en bosques de pino-encino templada (Fig. 4A, Fig. 4B). El grupo dos, incluyó a registros de jaguarundi, ocelote y tigrillo en bosques tropicales caducifolios de la Sierra Madre del Sur con 7.6 


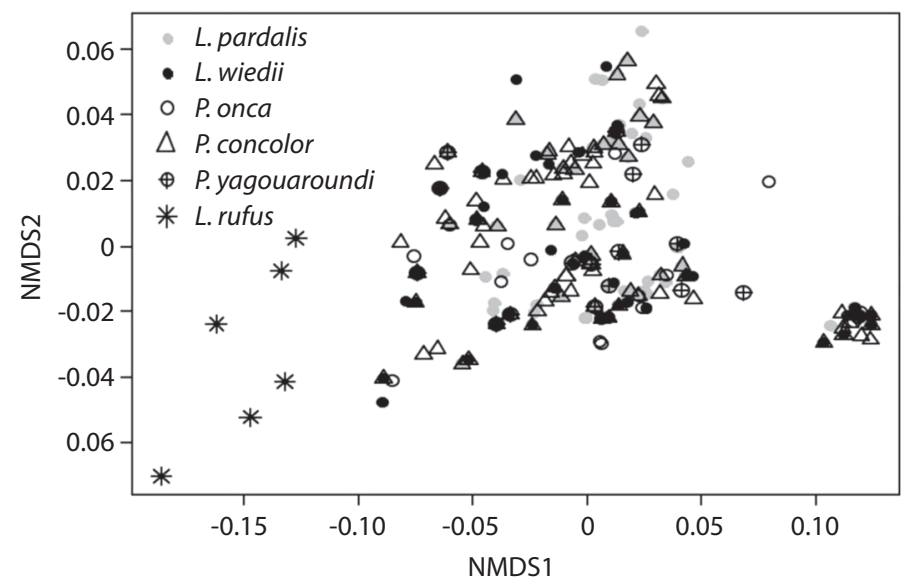

Fig. 3. Análisis de escalamiento multidimensional no métrico que muestra la distribución de los registros de felinos en un espacio en función de un conjunto de siete variables ambientales. Mientras que la mayoría de las especies muestran un importante traslape en su distribución, el lince (Lynx rufus) se distingue por ser el que muestra una mayor diferenciación en su distribución.

Fig. 3. Analysis of non-metric multidimensional scaling depicting the distribution of felid presence records in relation to a set of seven environmental variables. There is a general overlap in the distribution of the species with the exception of Lynx rufus which shows the most distinctive pattern.

registros por 100 días/cámara-trampa, terrenos agropecuarios en la Costa con 0.8 registros por 100 días/cámara-trampa y bosques de pino en el Eje Neovolcánico con 1.7 registros por 100 días/cámara-trampa (Fig. 4A, Fig. 4B). El grupo tres incluyeron los registros de puma, ocelote, tigrillo y jaguar para cuatro áreas: bosque tropical caducifolio del Eje Neovolcánico con 6.2 registros por 100 días/cámara-trampa, usos agropecuarios del Bajo Balsas con 3.1 registros por 100 días/cámara-trampa, bosques de pino de la Sierra madre del Sur con 1.5 registros por 100 días/cámara-trampa y bosque tropical subcaducifolio de la Costa con 2.6 registros por 100 días/cámara-trampa (Fig. 4A, Fig. 4B). El grupo cuatro incluyó únicamente a los bosques de encino de la Sierra Madre del Sur, donde se presentaron tasa de captura de $2.5,7.5$ y 2.5 registros por 100 días/cámaratrampa para puma, jaguarundi y ocelote, respectivamente (Fig. 4A, Fig. 4B). Por último, el quinto grupo incluyó a los dos registros de cámara-trampa de lince, los cuales se obtuvieron en los bosques tropicales caducifolios del Bajío, con una tasa de captura de 3.3 registros por 100 días/cámara-trampa (Fig. 4A, Fig. 4B).
Sólo 44 de los 701 registros correspondieron a sitios dentro de alguna de las 32 áreas naturales protegidas de carácter estatal y 13 de carácter federal existentes en la región. Prácticamente, la totalidad de estos registros se presentaron dentro de la Reserva de la Biosfera de Zicuirán Infiernillo (RBZI), y correspondieron a: $P$. onca $(\mathrm{n}=1), P$. concolor $(\mathrm{n}=22), P$. yagouaroundi $(\mathrm{n}=3)$, L. pardalis $(\mathrm{n}=15) \mathrm{y}$ $L$, wiedii $(\mathrm{n}=2)$. L. wiedii también se registró en el Parque Nacional Barranca del Cupatitzio. El lince fue la única especie para la que no se obtuvieron registros en alguna área protegida.

\section{DISCUSIÓN}

El trabajo de campo desarrollado en los últimos 10 años permitió pasar de 29 a 672 registros de felinos en la región de estudio, lo que significa un incremento de más del $2000 \%$ en la información disponible. A partir de esta información es posible tener una visión más completa de los patrones de distribución de las especies de felinos en esta importante porción de la costa del Pacífico mexicano. 

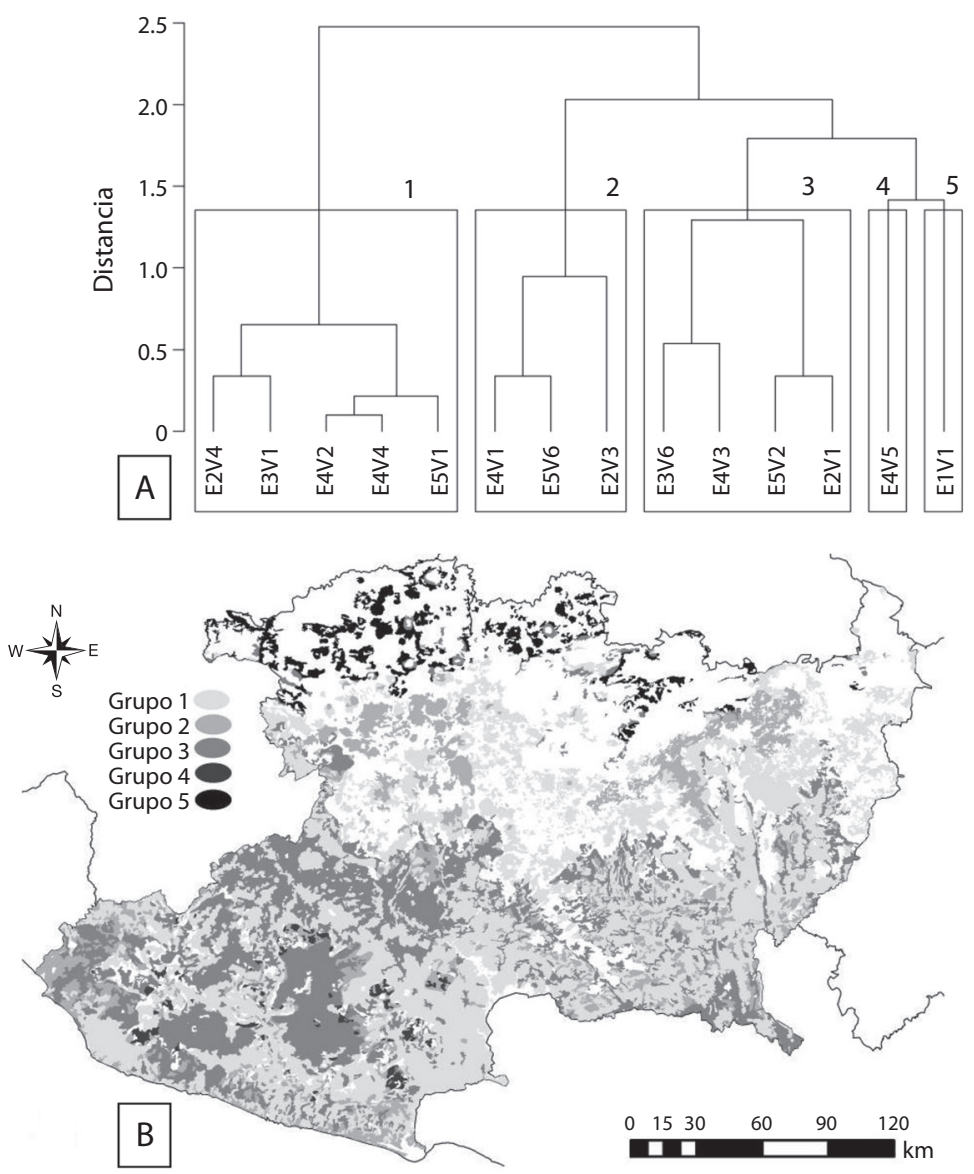

Fig. 4. A) Análisis de conglomerados que indica que se pueden distinguir cinco grupos de áreas con distintas combinaciones de cobertura vegetal y ecorregión, con base en los registros de cámara-trampa obtenidos para las distintas especies (simbología se detalla en métodos). B) Localización dentro del área de estudio de los cinco grupos identificados.

Fig. 4. A) Cluster analysis showing that it is possible to distinguish five groups of areas having different combinations of vegetation types and eco-regions based on the camera-trap records of the different felid species (key for different combinations of eco-regions and vegetation types is explained in the methods section). B) Geographic location of the five clusters.

Los resultados muestran que las cinco especies de felinos de afinidad tropical presentan una distribución relativamente extensa que abarca un amplio intervalo altitudinal (desde el nivel del mar hasta los $1760 \mathrm{msnm}$ ) y de diversos tipos de vegetación (bosques tropicales caducifolios y subcaducifolios, bosques de pino, bosques de encino y bosques templados mixtos). En contraste, la distribución del lince se restringió a los bosques más fríos, localizados entre 2000 y $2800 \mathrm{msnm}$, una situación similar a la reportada en regiones cercanas como el estado de Oaxaca (Monroy \& BrionesSalas, 2012).

Si bien hubo una alta coincidencia en los patrones de distribución de los felinos de afinidad tropical, existió también variación entre especies. Por ejemplo, mientras que alrededor del $40 \%$ de los registros del puma, ocelote y jaguarundi se presentaron en el bosque tropical caducifolio, solo el $30 \%$ de los registros de tigrillo, y alrededor del $20 \%$ de los de jaguar, se obtuvieron en este hábitat. Algo similar se observó en el caso del bosque de pino-encino, 
en donde existieron registros de todas las especies, siendo el ocelote la especie con el mayor porcentaje de registros con $57 \%$, seguido del puma con $27 \%$, el tigrillo con $12 \%$, el jaguarundi con $2.5 \%$, el jaguar con $1 \%$ y lince con $0.5 \%$. Esto sugiere la existencia de factores que operan a escalas más finas que pueden influir de manera diferencial sobre las preferencias de los felinos por distintos tipos de hábitat. Por ejemplo, la importancia de los bosques templados de encino-pino de la región para los felinos de afinidad tropical, ocelote, tigrillo, jaguarundi y jaguar, puede deberse a que proporcionan recursos claves durante la época seca (p. ej., agua, alimento o refugio) en la que estos se vuelven muy escasos en las zonas más cálidas y secas. Esta posibilidad adquiere mayor apoyo cuando se considera que los registros de las cámaras-trampa obtenidos en esos bosques correspondieron, principalmente, a la época seca del año, y que existe evidencia que muestra que los manantiales son sitios donde se concentra la actividad de los felinos y sus presas en la región (Charre-Medellín, 2012).

En este sentido, es de destacar que el jaguarundi fue la especie cuyos registros se distribuyeron en el ámbito más amplio de variación climática a pesar de presentar un número reducido de registros. En contraste, el ocelote, a pesar de ser la especie con el mayor número de registros, presentó una concentración de los mismos en un intervalo de amplitud climática muy reducido. Por otra parte, algunos de los registros de jaguarundi, tigrillo y ocelote obtenidos en este estudio corresponden a las localidades más áridas y calientes en las que se ha registrado la presencia de poblaciones de esas especies de felinos en México. De esta manera los resultados de este estudio permiten tener una visión más amplia sobre la adaptabilidad de los felinos para habitar en un amplio ámbito de condiciones climáticas (Torres-Romero, 2009; Chávez-Tovar, 2010).

No sólo las condiciones climáticas determinan la abundancia y distribución de las especies. Para los felinos de mayor tamaño como el jaguar y puma, se ha documentado que prefieren hábitats con cobertura vegetal densa, poco fragmentada, distante de caminos, cercanos a cuerpos de agua y mínima perturbación humana en general (Thatcher, Van Manen, \& Clark, 2006; Laundré, Loredo, Hernández, \& Núñez, 2009; Charre-Medellín, 2012). En concordancia con esto, en este estudio se encontró que la distribución y la mayor cantidad de registros de puma y jaguar coincidieron con las áreas con una mayor extensión continua de bosques tropicales y templados, poca densidad humana y disponibilidad de presas (Charre-Medellín, 2012). Por otra parte, los registros obtenidos en sitios agropecuarios, correspondieron principalmente a casos en los que los habitantes locales reportaban felinos acechando tanto al ganado o a aves de corral. Un estudio más detallado sobre el efecto del impacto humano en las poblaciones de felinos, puede servir para entender con mayor detalle cómo la perturbación humana interactúa con el clima y la escasez de recursos claves, como el agua, que pueden influir en los patrones de distribución y abundancia de los felinos, en un sistema altamente heterogéneo como el presente en el occidente de México.

Por otra parte, conocer la distribución de las especies a nivel regional, es el primer paso para implementar acciones de conservación, que permitan la persistencia de las poblaciones locales de especies, principalmente, aquellas que por sus requerimientos son más vulnerables a la extinción local, caso es el de los carnívoros en general y felinos en particular (Kanagaraj, Wiegand, Mohamed, \& Kramer-Schadt, 2013). En el área de estudio, urge contar con este tipo de información porque los bosques tropicales y bosques templados están actualmente afectados por elevadas tasas de deforestación anual de 1 y $1.8 \%$, además de una tasa común de degradación del $20 \%$ en la extensión total de cubiertas de bosques y selvas (Bocco et al., 2001; Trejo \& Dirzo, 2000; Mas-Caussel, Velásquez-Montes, \& Fernández-Vargas, 2005).

A esto se agrega la baja cobertura de áreas protegidas en las regiones costeras y la Sierra Madre del Sur, donde los resultados indican que se encuentra la mayor cantidad de registros de felinos. El presente estudio, sumado 
a posteriores iniciativas, que continúen documentando la presencia de felinos en zonas poco exploradas de la costa del Pacífico, son particularmente necesarias para delimitar nuevas áreas de conservación de felinos en: áreas del Bajo Balsas que colindan con el Estado de México y Guerrero en el Este, los bosques secos del Noroeste del Bajío, Este del Eje Neovolcánico y la Sierra Madre del Sur. Estas nuevas coberturas permitirían incrementar la conectividad entre poblaciones, para establecer una red con las pocas áreas naturales protegidas existentes del centro y occidente del país como Jalisco y el Estado de México (Monroy-Vilchis, Sánchez, Aguilera-Reyes, Suarez, \& Urios, 2008; Aranda, Botello, \& López-de Buen, 2012). De esta manera, la exploración de todas estas áreas, permitirá seguir incrementando el conocimiento sobre los factores que determinan los patrones de distribución de felinos a lo largo de gradientes ecológicos, permitiendo contar con mayores elementos para enfrentar el impacto humano actual y amenazas futuras como el cambio climático, el cual se anticipa tendrá un impacto significativo en la región al aumentar la temperatura promedio $\left(3.6^{\circ} \mathrm{C}\right)$ y disminuir la precipitación anual promedio (-7.8\%) en las próximas décadas (Sáenz-Romero et al., 2010; Sáenz-Romero et al., 2012)

\section{AGRADECIMIENTOS}

Agradecemos el apoyo en campo brindado por estudiantes del Laboratorio de Ecología de Vertebrados Terrestres Prioritarios. CharreMedellín agradece a CONACYT por la beca otorgada (239248), a la familia CamorlingaTorres, Manuel Barragán, Eduardo Soto y al ejido del Platanar, por su apoyo en el trabajo de campo. Este trabajo fue financiado por la Coordinación de Investigación Científica de la Universidad Michoacana de San Nicolás de Hidalgo (UMSNH), Fondos Mixtos CONACYT-Estado de Michoacán (Proyecto 41168) y el PIFI. SEMARNAT proporcionó el permiso para recolección científica (FAUT-0170) a nombre de Livia León Paniagua y la Facultad de Biología de la UMSNH otorgó las facilidades para la preparación del manuscrito.

\section{RESUMEN}

El estado de Michoacán se caracteriza por presentar una importante heterogeneidad ambiental, en términos de clima, topografía y tipos de vegetación, que incluyen al bosque tropical seco que se encuentra en peligro de extinción a nivel mundial. Algunos trabajos mencionan la presencia de las seis especies de felinos que habitan en México, para la región; sin embargo, la evidencia para apoyar estos trabajos es escasa, por lo que llenar esta falta de información es especialmente crítico en el caso de especies o hábitats amenazados. El objetivo de este estudio fue sistematizar la información y analizar los patrones de distribución de los felinos en el estado de Michoacán, dentro del centro-occidente de México. Realizamos una revisión de la información bibliográfica y contenida en bases de datos sobre la presencia de felinos en esta región. Asimismo, realizamos trabajo de campo que en el curso de diez años donde se aplicaron distintos métodos para detectar la presencia de especies de felinos (recorrido de senderos para obtener evidencia directa e indirecta de la presencia de las especies, trampas cámara y entrevistas). Localizamos 29 registros de presencia en literatura y bases de datos. Por otra parte acumulamos un total de $1107.5 \mathrm{~km}$ de transectos recorridos y 8699 días/cámara-trampa. A través de este esfuerzo de muestreo, generamos 672 registros de presencia de las seis especies. Lynx rufus fue la especie con menos registros totales $(n=3)$ y cuya distribución contrastó más con la del resto de las especies. La especie con más registros fue Leopardus pardalis $(\mathrm{n}=343)$. En general, el $89 \%$ de los registros de felinos se obtuvieron por debajo de los $1000 \mathrm{msnm}$. En promedio, la temperatura media anual y la precipitación anual donde se ubicaron los registros fue de $24^{\circ} \mathrm{C}$ y $1040 \mathrm{~mm}$ respectivamente. La especie que mostro un patrón más claro en términos de temperatura y precipitación fue L. rufus $\left(15.8 \pm 1.3^{\circ} \mathrm{C}\right.$ y $\left.941 \pm 171 \mathrm{~mm}\right)$. De acuerdo con el dendrograma resultante del análisis de conglomerados de las combinaciones de ecorregiones y tipos de vegetación se logró distinguir cinco grupos. Este estudio permite tener un panorama más completo de los patrones de distribución de los felinos en una región de importante contraste ambiental y sujeto a un fuerte impacto por las actividades humanas. Asimismo, los datos generados en este estudio pueden ayudar a profundizar nuestro entendimiento de la relación que existe entre la distribución de especies de felinos y las características del ambiente y servir como base para el desarrollo de estrategias de conservación local y regional.

Palabras clave: cámara-trampa, heterogeneidad ambiental, preferencias de hábitat, ecología de mamíferos silvestres. 


\section{REFERENCIAS}

Álvarez, T., Arroyo-Cabrales, J., \& González-Escamilla, M. (1987). Mamíferos (excepto Chiroptera de la costa de Michoacán, México). Anales de la Escuela Nacional de Ciencias Biológicas, 31, 13-62.

Álvarez-Solórzano, T. \& López-Vidal, J. C. (1998). Biodiversidad de los mamíferos en el Estado de Michoacán. Instituto Politécnico Nacional. Escuela Nacional de Ciencias Biológicas. Base de datos SNIB2010CONABIO proyecto No. P020. México, D. F.

Aranda, M. (2000). Huellas y otros rastros de los mamiferos grandes y medianos de México. Xalapa, Veracruz, México: Instituto de Ecología, A.C.

Aranda, M., Botello, F., \& López-de Buen, L. (2012). Diversidad y datos reproductivos de mamíferos medianos y grandes en el bosque mesófilo de montaña de la Reserva de la Biosfera Sierra de Manantlán, Jalisco-Colima, México. Revista Mexicana de Biodiversidad, 83, 778-784. doi: http://dx.doi.org/10.7550/ rmb. 24850

Baillie, J. E. M., Griffiths, J., Turvey, S. T., Loh, J., \& Collen, B. (2010). Evolution Lost: Status and Trends of the World's Vertebrates. United Kingdom: Zoological Society of London.

Bárcenas, H., \& Medellín, R. (2010). Ocelot (Leopardus pardalis) in Aguascalientes, México. The Southwesten Naturalist, 55(3), 447-449. doi: 10.1894/CLG-28.1

Bocco, G., Mendoza, M., \& Masera, O. R. (2001). La dinámica del cambio de uso de suelo en Michoacán. Una propuesta metodológica para el estudio de los procesos de deforestación. Boletín de Investigaciones Geográficas, 44, 18-38.

Borcard, D., Gillet, F., \& Legendre, P. (2011). Numerical Ecology with R. Use R! series. Springer, NY.

Botello, F., Monroy, G., Illoldi-Rangel, P., Trujillo-Bolio, I., \& Sánchez-Cordero, V. (2007). Sistematización de imágenes obtenidas en fototrampeo, una propuesta de ficha. Revista Mexicana de Biodiversidad, 78, 207210. doi: http://dx.doi.org/10.7550/rmb.5306

Brand, D. (1960). Coalcomán and Motines del Oro. An exdistrito of Michoacán, México. The Hague: Martinus Nijhoff. Austin, Texas: Institute of Latin American Studies, University of Texas.

Carranza-González, E. (2005). Vegetación. In L. E. Villaseñor-Gómez (Ed.), La biodiversidad en Michoacán: Estudio de Estado (pp. 38-45). México: Comisión Nacional para el Conocimiento y Uso de la Biodiversidad, Secretaría de Urbanismo y Medio Ambiente, Universidad Michoacana de San Nicolás de Hidalgo.

Ceballos, G., \& Ehrlich, P. R. (2002). Mammal population losses and the extinction crisis. Science, 296, 904 907. doi: 10.1126/science.1069349
Ceballos, G., List, R., Medellín, R., Bonacic, C., \& Pacheco, J. 2010a. Los felinos de américa. Cazadores sorprendentes. México: TELMEX. Retrieved from http://contenidosabiertos.academica.mx/jspui/ handle/987654321/130

Ceballos, G., García, A., Salazar, I., \& Espinoza, E. (2010b). Conservación de los vertebrados de selvas secas: patrones de distribución, endemismo y vulnerabilidad. In G. Ceballos, L. Martínez, A. García, E. Espinoza, J. Bezaury-Creel, \& R. Dirzo (Eds.), Diversidad, amenazas y áreas prioritarias para la conservación de las selvas secas del Pacifico de México (pp. 369-386). México: FCE - CONABIO - CONANPWWF- MÉXICO - ECOCIENCIA S.C. - TELMEX.

Charre-Medellín, J. F. (2012). Uso de manantiales por los mamiferos silvestres en bosques tropicales de Michoacán (Tesis de maestría). Universidad Michoacana de San Nicolás de Hidalgo, Morelia, Michoacán, México.

Chávez-León, G. \& Zaragoza-Ribera, S. (2009). Riqueza de mamíferos del Parque Nacional Barranca del Cupatitzio, Michoacán, México. Revista Mexicana de Biodiversidad, 89, 95-104. doi: http://dx.doi. org/10.7550/rmb.5414

Chávez-Tovar, J. C. (2010). Ecología y conservación del jaguar (Panthera onca) y puma (Puma concolor) en la región de Calakmul y sus implicaciones para la conservación de la Península de Yucatán (Tesis de doctorado). Universidad Nacional Autónoma de México. México, D.F.

CONANP (Comisión Nacional de Áreas Naturales Protegidas). (2013). Áreas Naturales protegidas Federales. Recuperado de http://sig.conanp.gob.mx/website/ pagsig/informacion/info.htm.

Cuervo-Robayo, A. P. \& Monroy-Vilchis, O. (2012). Distribución potencial del jaguar Panthera onca (Carnivora: Felidae) en Guerrero, México: persistencia de zonas para su conservación. Revista de Biología Tropical, 60(3), 1357-1367. doi: http://dx.doi. org/10.15517/rbt.v60i3.1813

Emmons, L. H., \& Feer, F. (1997). Neotropical Rain Forest Mammals, a Field Guide. 2nd ed. The University of Chicago Press.

ESRI. (2008). ArcGis reléase Versión 9.3. Redlands, California, USA: Environmental Scientific Research Institute.

Garduño-Monroy, V. (2005). El Relieve, Descripción física y biótica (Cap. 2). In L. E. Villaseñor-Gómez (Ed.), La biodiversidad en Michoacán: Estudio de Estado (pp. 21-24). México: Comisión Nacional para el Conocimiento y Uso de la Biodiversidad, Secretaría de Urbanismo y Medio Ambiente, Universidad Michoacana de San Nicolás de Hidalgo. 
INEGI (Instituto Nacional de Estadística y Geografía). (2013a). Continúo de Elevaciones Mexicano 3.0. Recuperado de http://www.inegi.org.mx/geo/contenidos/datosrelieve/continental/Descarga.aspx.

INEGI (Instituto Nacional de Estadística y Geografía). (2013b). Carta de Uso del Suelo y Vegetación, Serie $V$, escala 1: 250 000. México.

Jiménez-Maldonado, R. E. (2007). Modelación de nicho ecológico y coexistencia de ocelotes (Leopardus pardalis) y gatos monteses (Lynx rufus) en México (Tesis de maestría). Universidad Autónoma de Querétaro. México.

Kanagaraj, R., Wiegand, T., Mohamed,A., \& Kramer-Schadt, S. (2013). Modelling species distributions to map the road towards carnivore conservation in the tropics. The Raffles Bulletin of Zoology, 28, 85-107. http:// zoobank.org/urn:1sid:zoobank.org:pub:43A334A0CECD-4916-BBF2-B572A95CFEE4

Laundré, J. W., Loredo, J., Hernández, L., \& Núñez, D. (2009). Evaluating potential factors affecting puma Puma concolor abundance in the Mexican Chihuahuan Desert. Wildlife Biology, 15, 207-212. doi: http://dx.doi.org/10.2981/07-077

León-Paniagua, L. (1999). Computarización de las colecciones del museo de Zoología Alfonso L. Herrera para su incorporación a la REMIB: Fase I. Facultad de Ciencias, Universidad Nacional Autónoma de México. NIB2010-CONABIO proyecto No. J123. México, D.F.

Leopold, A. (1959). Wildlife of Mexico: The game birds and mammals. University of California Press, Berkeley.

López-Wilchis, R. (1998). Base de datos de mamíferos de México depositados en colecciones de Estados Unidos y Canadá. Universidad Autónoma Metropolitana. Unidad Iztapalapa. Bases de datos SNIB-CONABIO. Proyecto No. P130. México, D.F.

Mas-Caussel, J. F., Velásquez-Montes, A., \& FernándezVargas, T. (2005). Monitoreo de los cambios de cobertura del suelo en Michoacán. In L. E. Villaseñor-Gómez (Ed.), La biodiversidad en Michoacán: Estudio de Estado (pp. 204-206). México: Comisión Nacional para el Conocimiento y Uso de la Biodiversidad, Secretaría de Urbanismo y Medio Ambiente, Universidad Michoacana de San Nicolás de Hidalgo.

Monroy, G. \& Briones-Salas, M. (2012). Primeros datos sobre Área de actividad de gato montés (Lynx Rufus) en Oaxaca, México. Acta Zoológica Mexicana, 28(2), 471-474.

Monroy-Vilchis, O., Sánchez, O., Aguilera-Reyes, U., Suarez, P., \& Urios, V. (2008). Jaguar (Panthera onca) in the State of Mexico. The Southwestern Naturalist, 53, 533-537.

Monroy-Vilchis, O., Zarco-González, M., Rodríguez-Soto, C., Soria-Díaz, L., \& Urios, V. (2011). Fototrampeo de mamíferos en la Sierra Nanchititla, México: abundancia relativa y patrón de actividad. Revista de Biología Tropical, 59(1), 373-383.

Murray, J. L., \& Gardner, G. L. (1997). Leopardus pardalis. Mammalian Species, 548, 1-10.

Núñez, R. (2007). Distribución y situación del jaguar en el occidente de México. In G. Ceballos, \& R. List (Eds.), Conservación y manejo del jaguar en México: estudios de caso y perspectivas (pp.25-40). México: CONABIO-Alianza WWF/Telcel-Universidad Nacional Autónoma de México.

Núñez, R. (2012). The status of jaguars in Michoacán, Mexico. Wild Felid Monitor, 4, 18.

Olalde-García, G. (1997). Mamíferos de talla corporal mediana y grande de tres localidades en el estado de Michoacán (Tesis de licenciatura). Universidad Michoacana de San Nicolás de Hidalgo, Morelia, Michoacán, México.

Oliveira, T. (1998). Leopardus wiedii. Mammalian Species, 579, 1-6.

Olson, D. M., Dinerstein, E., Wikramanayake, E. D., Burgess, N. D., Powell, J. V. N., Underwood, E. C., \& Kassem, K. R. (2001). Terrestrial Ecoregions of the World: A New Map of Life on Earth. BioScience, $51,933-938$

R Core Team. (2013). R: A language and environment for statistical computing. R Foundation for Statistical Computing, Vienna, Austria.

Sáenz-Romero, C., Rehfeldt, G. E., Crookston, N. L., Duval, P., St-Amant, R., Beaulien, J., \& Richardson, B. A. (2010). Spline models of contemporary, 2030, 2060 and 2090 climates for Mexico and their use in understanding climate-change impacts on the vegetation. Climatic Change, 102, 595-623. doi: 10.1007/ s10584-009.9753-5

Sáenz-Romero, C., Rehfeldt, G. E., Crookston, N. L., Duval, P., St-Amant, R., \& Beaulien, J. (2012). Spline models of contemporary, 2030, 2060 and 2090 climates for Michoacán state, México. Impacts on the vegetation. Revista Fitotecnia Mexicana, 35(4), 333-345.

Silver, S., Ostro, E. T., Marsh, L. K., Maffei, L., Noss, A. J., Kelly, M. J., \& Ayala G. (2004). The use of camera traps for estimating jaguar Panthera onca abundance and density using capture/recapture analysis. Oryx, 38(2), 1-7. doi: http://dx.doi.org/10.1017/ S0030605304000286

Thatcher, C. A., Van Manen, F. T., \& Clark, J. D. (2006). Identifying suitable sites for Florida panther reintroduction. Journal of Wildlife Management, 70, 752763. doi: http://dx.doi.org/10.2193/0022-541X(2006 )70[752:ISSFFP]2.0.CO;2

Torres-Romero, E. J. (2009). Densidad, abundancia, uso de hábitat y patrones de actividad del ocelote 
(Leopardus pardalis) en la zona noreste del Estado de Quintana Roo: Estudio usando cámaras trampa (Tesis de maestría). El Colegio de la Frontera Sur, México.
Trejo, I., \& Dirzo, R. (2000). Deforestation of seasonally dry tropical forest: a national and local analysis in Mexico. Biological Conservation, 94, 133-142. doi: 10.1016/S0006-3207(99)00188-3.

\section{APÉNDICE 1}

Distribución de los registros de presencia de felinos en relación a los gradientes de temperatura media anual y precipitación anual presentes en área de estudio

\section{APPENDIX 1}

Distribution of presence records of felids in relation to mean average temperature and annual precipitation

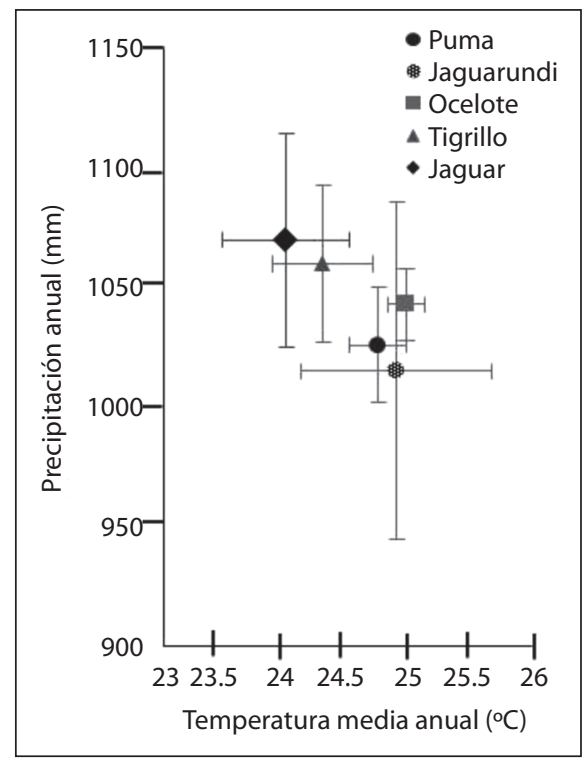

Los valores mostrados corresponden a los promedios de cada especie y las barras de error corresponden a sus intervalos de confianza del $95 \%$.

Values correspond to the species averages, error bars correspond to $95 \%$ confidence intervals. 
